\title{
Ventricular fibrillation due to coronary spasm at the site of myocardial bridge - A case report-
}

\author{
Jung Gi Choi, Cheon Hee Park, Cheol Seung Lee, and June Seog Choi \\ Department of Anesthesiology and Pain Medicine, Gwangju Christian Hospital, Gwangju, Korea
}

\begin{abstract}
Myocardial bridge is a congenital anomaly characterized by narrowing of some of the epicardial coronary arterial segments running in the myocardium during systole. Occasionally, the compression of a coronary artery by a myocardial bridge can be associated with the clinical manifestations of myocardial ischemia, and might even trigger a myocardial infarction or malignant ventricular arrhythmias. We report a case of ventricular fibrillation due to coronary spasm at the site of myocardial bridge. A 56-year-old man who had suffered from bronchial asthma was given remifentanil combined with sevoflurane in general anesthesia for endoscopic sinus surgery. During the surgery, ventricular fibrillation occurred following coronary spasm with bradycardia, hypotension, bronchospasm. we found myocardial bridge that coincided with an area of coronary spasm after coronary angiography. (Korean J Anesthesiol 2010; 58: 99 103)
\end{abstract}

\section{Key Words: Coronary spasm, Myocardial bridge, Remifentanil, Ventricular fibrillation.}

Typically, most of the coronary artery rests on the epicardium but some cases, the epicardial coronary artery runs congenitally into the myocardium. During the systole, the heart muscle exerts pressure across the region and constricts the coronary artery, which is called the myocardial bridge (MB) [1]. Since coronary angiography is a common procedure and non-invasive methods, such as multidetector computed tomography (MDCT), can make a diagnosis of even a minute part more simple, myocardial bridges have been found in a substantial number of patients, even though the prevalence varies according to the report [2]. Most clinical findings show that the MB does not lead to major problems while some cases report that unstable perfusion of the coronary arteries can cause serious cardiac disorders, such as myocardial ischemia, myocardial infarction, arrhythmia, and even sudden cardiac death $[3,4]$. Therefore, the clinical importance of MB is increasing. During general anesthesia, the authors experienced a bronchial spasm after an unexpected elevation of the ST segment, followed by ventricular fibrillation immediately after sudden bradycardia and hypotension,

Received: July 21, 2009. Revised: 1st, August 9, 2009; 2nd, August 23, 2009. Accepted: October 1, 2009.

Corresponding author: Cheon Hee Park, M.D., Department of Anesthesiology and Pain Medicine, Gwangju Christian Hospital, 264, Yangnimdong, Nam-gu, Gwangju 503-715, Korea. Tel: 82-62-650-5154, Fax: 82-62-671-7447, E-mail: pch1962@hanmail.net

@) This is an open-access article distributed under the terms of the Creative Commons Attribution Non-Commercial License (http:// creativecommons.org/licenses/by-nc/3.0/), which permits unrestricted non-commercial use, distribution, and reproduction in any medium, provided the original work is properly cited. 
which was suspected to be a coronary spasm. After surgery, coronary arteriography detected a MB that could cause a coronary spasm. We report this case with a review of the relevant literature.

\section{Case Report}

A 56-year-old, male patient $(62 \mathrm{~kg}$ ) with sinusitis presented for the removal of a nasal polyp and endoscopic sinus surgery. The patient had been administered acebrophylline, montelukast sodium orally for bronchial asthma, and was using formoterol fumarate dihydrate micronized budesonide turbuhaler every morning for the last two years. He often felt pain and palpation on the chest when he coughed heavily but the electrocardiogram (ECG) and echocardiography tests did not reveal any specific abnormalities. Twenty four hour Holter monitoring had been advised to keep a log of the heart's electrical activity. However, the patient had not received further medical treatments because the symptoms had disappeared. He reported a feeling of anxiety when he was hospitalized, but there were no abnormalities in the blood test, simple chest X-rays, electrocardiogram and echocardiography. For administration before surgery, famotidine $20 \mathrm{mg}$ and glycopyrrolate $0.2 \mathrm{mg}$ were injected into the muscle site, and on the morning of the day of surgery, salbutamol sulfate was used as a spray. Upon admission, the vital signs indicated that the blood pressure, heart rate and oxygen saturation was 100/60 mmHg, 68 beats/min and $99 \%$, respectively. Patient monitoring was measured with a non-invasive auto blood pressure, pulse oximetry, and electrocardiogram. For the induction of anesthesia, propofol $2 \mathrm{mg} / \mathrm{kg}$ and rocuronium $0.6 \mathrm{mg} / \mathrm{kg}$ were administered and endotracheal intubation was performed after muscle relaxation had been fully achieved. The blood pressure and heart rate immediately after endotracheal intubation was 140/90 $\mathrm{mmHg}$ and 90 beats/min, respectively. For the maintenance of anesthesia, sevoflurane 2-3.0 vol\%, $\mathrm{O}_{2} 1$ $\mathrm{L} / \mathrm{min}$, and air $2 \mathrm{~L} / \mathrm{min}$ were administered, and controlled respiration was performed to set the PET $\mathrm{CO}_{2}$ (partial pressure of end-tidal carbon dioxide) to $30-35 \mathrm{mmHg}$. Remifentanil was administered from $0.25 \mu \mathrm{g} / \mathrm{kg} / \mathrm{min}$ at the onset but reduced to $0.05 \mu \mathrm{g} / \mathrm{kg} / \mathrm{min}$ at the end in a stepwise manner. After the induction of anesthesia, $200 \mathrm{mg}$ of isepamicin was administered and the surgeon added a aqueous solution of lidocaine-epinephrine $(1: 10,000)$ into a cotton roll and placed it into the patient's nasal cavity. After pulling it out 5 minutes later, a solution of lidocaine-epinephrine (1:100,000) was injected into the membrane, and the operation was started. For his vital signs during surgery, the blood pressure range, heart rate range was $90-110 / 55-66 \mathrm{mmHg}$ and $60-70$ beats/ min, respectively, and the electrocardiogram showed regular signs while the oxygen saturation and end-tidal $\mathrm{CO}^{2}$ pressure was $99-100 \%$ and $35 \mathrm{mmHg}$, respectively.

Five minutes after the operation was started, a minor abnormality of a ST segment elevation appeared. After 10 minutes, the elevation of the ST segment increased along with a gradual decrease in heart rate. The plateau observed in capnography at the release of gas out of the lung was decreasing by degrees. An immediate change to manual control ventilation with 100\% oxygen was made, but there was a perceivable sense of resistance at the respiratory bag. The vital signs at that time were a blood pressure, heart rate oxygen saturation range and PET $\mathrm{CO}_{2}$ of $88 / 50 \mathrm{mmHg}, 50$ beats/min, 84-99\% and 45-48 mmHg, respectively. The vital signs at the subsequent measurements revealed a sheer decrease in BP and heart rate to $88 / 50 \mathrm{mmHg}$ and 40 beats/ min, respectively (Fig. 1). Immediately, ephedrine $8 \mathrm{mg}$ and atropine $0.5 \mathrm{mg}$ were administered. In 10 minutes, however, a ventricular fibrillation occurred and the surgeon's attention was called to cease the operation, followed by CPR. With manually controlled ventilation and external cardiac massage, administration of external $360 \mathrm{~J}$ shocks restored normal sinus rhythm. At that moment, the vital signs were a $\mathrm{BP}$, heart rate, oxygen saturation and $\mathrm{PET} \mathrm{CO}_{2}$ of $95 / 55 \mathrm{mmHg}$, 120 beats/min, 98\%, $52 \mathrm{mmHg}$, respectively. A catheter was then inserted into the radial artery for an arterial blood gas analysis ( $\mathrm{ABGA}$ ). The results of the arterial blood gas analysis were a $\mathrm{pH}, \mathrm{PaCO}_{2}, \mathrm{PaO}_{2}, \mathrm{HCO}_{3}{ }^{-}$and a $\mathrm{SaO}_{2}$ of 7.167, 58.7 $\mathrm{mmHg}, 371.1 \mathrm{mmHg}, 21.4 \mathrm{mM} / \mathrm{L}$ and $97.9 \%$, respectively. After consulting with the surgeon, we reached the conclusion that the operation should be terminated. The vital signs were maintained with blood pressure, heart rate oxygen saturation and PET $\mathrm{CO}_{2}$ of 100-110/60-65 mmHg, 110-120/ min, 98\%, $43 \mathrm{mmHg}$, respectively. The result of $A B G A$ indicated a $\mathrm{pH}, \mathrm{PaCO}_{2}, \mathrm{PaO}_{2}, \mathrm{HCO}_{3}{ }^{-}$and $\mathrm{SaO}_{2}$ of 7.281, 48.4 $\mathrm{mmHg}, 268.0 \mathrm{mmHg}, 23.0 \mathrm{mM} / \mathrm{L}$ and $99.1 \%$, respectively. At the termination of the operation, the patient was restored to spontaneous respiration and awakened. He was transported to the intensive care unit while being intubated with an endotracheal tube.

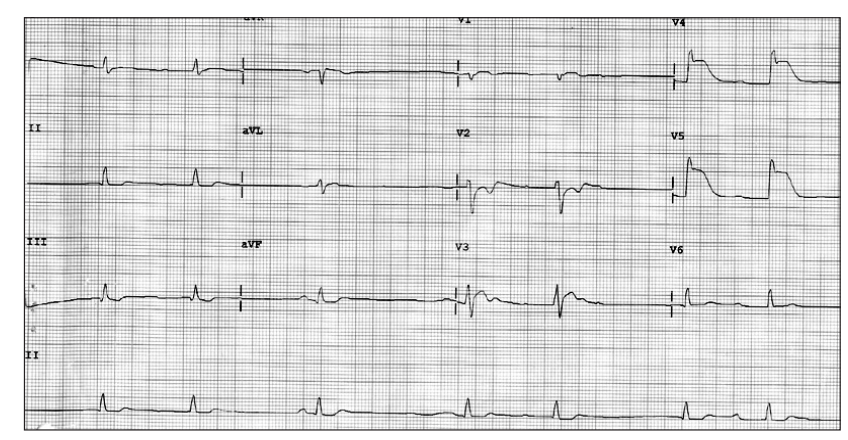

Fig. 1. Eletrocardiographic recordings before ventricular fibrillation in this case. The ECG shows ST segment elevation in lead V3-V5. 

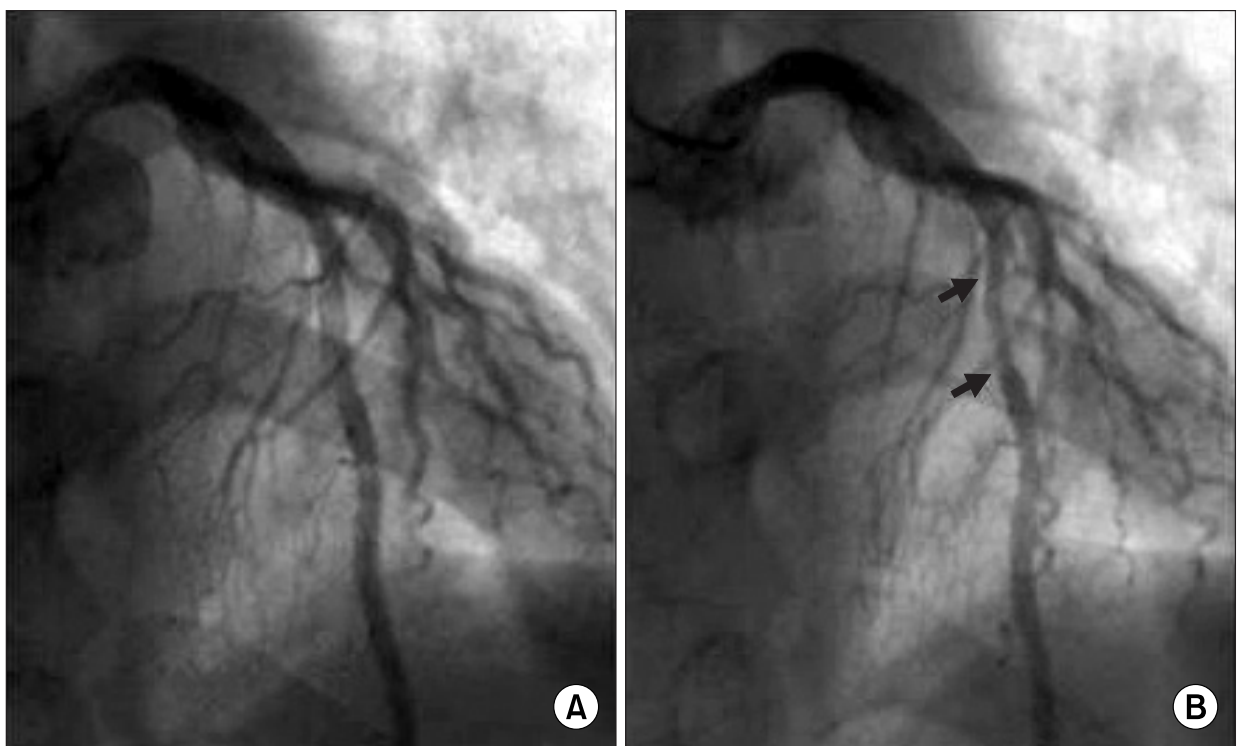

Fig. 2. Coronary angiogram showing marked systolic narrowing of the left anterior descending artery (A: Diastole, B: Systole)

The vital signs measured at the arrival to the intensive care unit showed a BP, $\mathrm{SpO}_{2}$ and heart rate of 110/65 mmHg, 99\% and 120 beats/min, respectively. The patient complained that he felt tight in the chest and at his request, salbutamol sulfate, a medication usually taken by him, was administered by spray. A slight elevation of the sinus rhythm and ST segment in the electrocardiogram was suspicious of myocardial ischemia, so nitroglycerine 1-2 $\mu \mathrm{g} / \mathrm{kg} / \mathrm{min}$ was administered continuously. The chest X-rays and echocardiography were normal, and the cardiac marker test demonstrated Troponin-T was positive. The Troponin-I level was $5.33 \mathrm{ng} / \mathrm{ml}$ (normal <0-0.1 ng/ $\mathrm{ml}$ ), and the CK-MB also was normal. For approximately 2 hours, the vital signs were maintained quiet normally with the $\mathrm{BP}$, heart rate and $\mathrm{SpO}_{2}$ at 95-100/50-55 mmHg, 90-110 beats/min and $99 \%$, respectively, when the ST segment elevation disappeared and the suffocating feeling in the chest had been ameliorated to some degree. After consulting with a cardiac physician, coronary angiography was used to determine if the patient's vital signs had returned to normal. A follow-up examination of the ECG kept showing a normal rhythm, and for 24 hours, the normal vital signs were maintained with no abnormal findings. Therefore, he was transferred to the general ward. The coronary angiography performed on the next day revealed no abnormal findings, such as an arteriosclerotic legion or thrombus (Fig. 2A), except for a myocardial bridge, approximately $2-2.5 \mathrm{~cm}$ in size, displaying 60-65\% vascular stenosis during systolic period at themiddle part of the left anterior descending artery, which corresponded to the ST segment elevation of $V_{3}-V_{5}$ (Fig. 2B). Three days later, when hospitalization care at the internal medicine ended, he was discharged home without any particular abnormalities under a follow-up observation.

\section{Discussion}

A myocardial bridge is a congenital coronary anomaly that is present when a segment of the epicardial coronary artery runs intramurally through the myocardim and the coronary artery is compressed with each systole [1]. The prevalence of a myocardial bridge (MB) varies. Recently, Leschka et al. [2] performed a comparative experiment of 100 patients with chest pain using conventional coronary angiography and multidetector computed tomography, and reported that coronary angiography detected MB in 12 (12\%) out of 100 patients, whereas MDCT detected MB in 26 patients (26\%). According to them, the reason why MDCT showed a higher depiction rate than conventional coronary angiography was that MDCT can diagnose the depth and length of MB, as well as the extent of systolic compression pressure more closely. In addition, the most common affected region of $\mathrm{MB}$ is the left anterior descending (LAD) artery, and the MB is usually distributed over the connecting area of the proximal and middle part of the LAD artery [2].

The clinical features vary from silence to atypical chest pain or angina symptoms according to the degree of systolic compression pressure or the depth and length of the tunneled segment. Although the ECGs of patients with MB usually show normal findings, stress testing might induce signs of ischemia, conduction disturbances, or arrhythmia, etc. [1]. In other words, the clinical results of patients with MB are relatively mild and do not bring up serious issues. However, $\mathrm{MB}$ is known to cause unstable angina pectoris, myocardial infarction, arrhythmia and even sudden death when the coronary artery perfusion is unstable [3,4].

There have been several studies on the mechanisms of 
ischemia caused by MB. However, their suggestions with vascular stenosis on the proximal part of MB or with the systolic compression could not give a satisfactory explanation to myocardial ischemia or patients' symptoms. Recently, Ge et al. [5] demonstrated with image analysis using intravascular ultrasound (IVUS) that the blood flow at the diastole in patients with MB increased in proportion to the occlusive time during the systole, and that the diastolic/systolic flow velocity ratio increased. They reported that the coronary vascular lumen at the late systole will be the minimum in patients with $M B$, while the vascular lumen at the late systole and early diastole will be a maximum in patients without. In addition, an increase in early diastolic flow triggers the still compressed narrow bridge segment, causing perfusion impairment in the coronary artery, which makes a crucial mechanism for ischemia. In particular, the occurrence of tachycardia reduces the filling time of the diastolic coronary and causes myocardial ischemia easily, whereas the increased systolic compression enhances the vascular compression during the diastole and systole, provoking the symptoms of MB easily. In addition, the hypofunction of endotheliocytes and angiospasm play an important role as a mechanism for myocardial ischemia $[6,7]$.

The present study showed that ventricular fibrillation occurred after coronary spasm during anesthesia. Coronary angiography performed after surgery did not reveal any fixed arteriosclerotic lesions, but found MB showing a stenosis in the coronary artery at the systole. A provocation test was not attempted during the coronary angiography because the patient underwent CPR. However, a myocardial bridge, approximately $2-2.5 \mathrm{~cm}$ in size, was found during the operation (Fig. 2B) displaying 60-65\% vascular stenosis during the systolic period at the middle part of the left anterior descending artery, which corresponded to the ST segment elevation of $\mathrm{V}_{3}-\mathrm{V}_{5}$ (Fig. 1). The MB is believed to be responsible for the coronary spasm during anesthesia.

Regarding the possibility of a coronary spasm at the site of MB triggering myocardial infarction, Gallat et al. [6] suggested that an ergonovine provocation test showed a positive response in the case of patients with MB who had a myocardial infarction without any arteriosclerotic coronary disorders on angiocardiography. In an animal experiment on pigs, Saitho et al. [7] reported that damage to the vascular endotheliocyte due to repeated coronary compression caused a coronary spasm. In a comparative experiment on the incidence of coronary spasm, Teragawa et al. [8] reported that among patients with chest pain, the incidence of coronary spasm was higher in patients with MB than in the patients without (30 out of 41 patients with MB [73\%] 29 out of 73 patients without [40\%]). Although there has not been an established theory regarding the mechanisms for $M B$, such as the high incidence of coronary spasm, histological studies suggested that arteriosclerotic lesions do not appear in patients with $\mathrm{MB}$ on the coronary angiography, but they may be suppressed in the MB region. Hence, the likelihood of coronary spasm at $\mathrm{MB}$ is high $[8,9]$. A coronary spasm also occurs when damage to the vascular smooth muscle and endotheliocyte causes a vascular malfunction, and repeated compression and shear stress to the $\mathrm{MB}$ are believed to worsen the vascular dysfunction $[7,8]$.

The gradual elevation of the ST segments concomitant with an increase in the air way pressure due to bronchoconstriction in the present case suggests that parasympathetic accentuation was developed by an inducing factor during general anesthesia, causing an imbalance in the automatic nervous system. It is believed that such parasympathetic accentuation first causes coronary spasm at MB showing an endothelial dysfunction in the coronary artery governed by the parasympathetic nervous system, while bronchial asthma also activated by parasympathetic accentuation causes bronchoconstriction, resulting in an increase in air way resistance. In an experiment study, Lee et al. [10] reported that the influence of remifentanil and nasal packing of epinephrine on the hemodynamic changes in endoscopic sinus surgery can accentuate the vagus nerve. Although the precise amount of epinephrine absorbed cannot be measured precisely because all the epinephrine packed in cotton does not infiltrate into the nasal cavity, it is suggested that the absorption of low doses (1-2 $\mu \mathrm{g} / \mathrm{min})$ will stimulate the vascular relaxant $\beta_{2}$ receptor to cause hypotension and bradycardia. In particular, the significantly high frequency of hypotension and bradycardia with $0.25 \mu \mathrm{g} / \mathrm{kg} / \mathrm{min}$ of remifentanil was reported to come from the side effects of blocking on vagus nerve stimulation and sympathetic activation.

In our case, where the vital signs during the operation did not change enough to cause tachycardia or any significant changes affecting the level of myocardial oxygen consumption or supply, such as hypothermia, hypoxia, etc, vagus nerves accentuation is believed to be a factor to make a MB cause a coronary spasm. Glycopyrrolate or ephedrine can be helpful to prevent bradycardia or hypotension, but they should be used with care because they can induce myocardial ischemia due to tachycardia in patients with MB.

Nitroglycerine, which is commonly used for ischemic heart diseases, was used in our case. According to Hongo et al. [11], the use of nitroglycerine for the treatment of coronary spasm in patients with MB can decrease the intrinsic tone of the coronary artery wall and aggravate the ischemia caused by MB due to positive inotrophy stimulated by the sympathetic nerve. However, Yu et al. [12] reported that among the medications used in a long-term clinical follow-up of patients with $M B$, the appropriate administration of a beta blocker, calcium channel blocker, and nitrate product according to 
the patient's condition showed favorable outcomes on most symptoms. In addition, Koji et al. [3] suggested that the use of nitroglycerine to treat ischemia was quite effective in patients with MB associated with a coronary artery spasm. This case also highlights the use of nitroglycerine to treat ischemia in patients suspected of having MB to attenuate the suffocating feeling of which the patients complained, and did not aggravate the myocardial ischemia. Therefore, more case reports and studies on the use of nitroglycerine will be needed. Moreover, in light of such cases, the use of nitroglycerin in patients with MB needs more monitoring with caution.

In general, a beta blocker and calcium channel blocker are used to secure hemodynamic balance during anesthesia for patients with MB $[12,13]$. However, some reports claimed that the calcium channel blocker is more effective in the treatment of coronary artery spasm in patients with MB than a beta blocker because a beta blocker decreases the systolic narrowing in a segment of $\mathrm{MB}$, aggravating the coronary artery spasm [3,8].

Regarding the choice of anesthetics, Lee et al. [14] used sevoflurane for patients with MB because there is a low occurrence of cardiac rate accentuation and no coronary steal. However, Iwama et al. [4] published a case report of a fatal acute myocardial infarction during general anesthesia in a child with $M B$, in which sevoflurane is regarded as a vasodilator to cause hypotension and lead to a sheer decrease in coronary blood flow. The choice of anesthetics for patients with $\mathrm{MB}$ also requires more clinical case results and further study.

In conclusion, cardiopulmonary resuscitation was performed due to sudden ventricular fibrillation that occurred after a progressive elevation of the ST segment in a patient with an undetected underlying disorder in the coronary artery. As a coronary spasm was suspected, coronary angiography was performed after surgery and a MB was found showing constriction at the systole. There were no other findings of fixed arteriosclerotic lesions or thrombus. Regarding the mechanisms for coronary artery spasm in patients with $M B$, an imbalance of the automatic nerve system and vascular over response have attracted attention. Therefore, a close diagnosis using the MDCT at the early phase should be made, and the management of peri-operative anesthesia for patients with an underlying disorder, such as $M B$, requires discreet judgments in anesthetic methods and medication use with more caution in monitoring the ECG and vital signs.

\section{References}

1. Möhlenkamp S, Hort W, Ge J, Erbel R. Update on myocardial bridging. Circulation 2002; 106 : 2616-22.
2. Leschka S, Koepfli P, Husmann L, Plass A, Vachenauer R, Gaemperli $\mathrm{O}$, et al. Myocardial bridging: depiction rate and morphology at CT coronary angiography-comparision with conventional coronary angiography. Radiology 2008; 246: 754-62.

3. Koji K, Norikatsu M, Yuji H, Yuji S, Mareomi H, Kunio H. Coronary vasospasm at the site of myocardial bridge. Angiology 1998; 49: 659-63.

4. Iwama H, Kaneko T, Watanabe K, Takasu M, Terada K, Sugiyama Y. Fatal acute myocardial infarction during general anesthesia in a 7-yr-old boy associated with total intramural coronary arteries. Anesthesiology 1997; 87: 426-9.

5. Ge J, Jeremias A, Rupp A, Abels M, Baumgart D, Liu F, et al. New signs characteristic of myocardial bridging demonstrated by intracoronary ultrasound and Doppler. Eur Heart J 1999; 20: 1707-16.

6. Gallat B, Adams C, Saudemont JP, Fruchaud J, Hiltgen M. Myocardial bridge of the left anterior descending coronary artery and myocardial infarction does coronary spasm play a part? Arch Mal Coeur Vaiss 1991; 84: 517-23.

7. Saitoh S, Muto M, Osugi T, Aikawa K, Matsumoto K, Onogi $F$, et al. Repeated epicardial coronary artery endothelial injuries lead to a global spontaneous coronary artery spasm. Coron Artery Dis 2004; 15: 137-45.

8. Teragawa H, Fukuda Y, Matsuda K, Hirao H, Higashi Y, Yamagata $T$, et al. Myocardial bridging increases the risk of coronary spasm. Clin Cardiol 2003; 26: 377-83.

9. Ishii T, Asuwa N, Muasuda S, Ishikawa Y. The effects of a myocardial bridge on coronary artherosclerosis and ischemia. J Pathol 1998; 185: 4-9.

10. Lee HM, Kim SY. Effects of remifentanil on hemodynamic of exogeneous epinephrine during endoscopic sinus surgery. Korean J Anesthesiol 2007; 52: 262-8.

11. Hongo Y, Tada H, Ito K, Yasumura Y, Miyatake K, Yamagishi M. Augmentation of vessel squeezing at coronarymyocardial bridge by nitroglycerin: Study of quantitative coronary angiography and intravascular ultrasound. Am Heart J 1999; 138: 345-50.

12. Yu HS, Jeong MH, Kim W, Shon IS, Kim KH, Hong YJ, et al. The long-term clinical follow-up of the patients with myocardial bridge. Chonnam Med J 2005; 41: 161-5.

13. Schwarz ER, Klues HG, Vom DJ, Klein I, Krebs W, Hanrath P. Functional, angiographic and intracoronary Doppler flow characteristics in symptomatic patients with myocardial bridging: effect of short-term intravenous beta-blocker medication. J Am Coll Cardiol 1996; 27: 1637-45.

14. Lee WK, Kim EJ, Lee JH, Lee SG, Ban JS, Min BW. Anesthetic management of unstable angina in a patient with myocardial bridge. Korean J Anesthesiol 2007; 53: 119-22. 